\title{
Analysis of the Motor NVH Based on Labview
}

\author{
Renyin Song \\ Ningde Normal University, Ningde, Fujian 352100 \\ hunter2011@foxmail.com
}

Keywords: Labview; Motor; NVH

\begin{abstract}
As motors are widely applied in the fields of industry, agriculture, etc., how to test, analyze and suppress motor vibration and noise, more and more people's attention. Virtual instrument technology, compared to traditional instruments with high performance, scalability and strong advantages in engineering and testing areas to be more widely used. Therefore, the combination of virtual instrument technology, establish test and analysis system motor noise and vibration is a viable solution. In this paper, the noise and vibration problems virtual instrument technology used in motors, the establishment of a major research paper is divided into three sections based on virtual instruments motor noise and vibration measurement and analysis system: The first two parts were studied hardware and software system components, the establishment of a complete hardware and software systems; third part of an experimental study of noise and vibration, to verify the correctness and effectiveness of the system.
\end{abstract}

\section{Introduction}

Noise and Vibration motors is very complex, it involves electromagnetic energy conversion, mechanical vibrations, special physical acoustics, electronics and mathematics in many disciplines. Many motor types, there are different specifications for each type of motor, type of construction and use, need to parse each motor part internal structure of the motor can be divided into the mechanical structure, circuit, magnetic circuit, insulation structure. Motor lubrication, ventilation, installation environment there are many specific requirements.

More difficult than the vibration of the motor noise study because of noise involved is roughly the overall effect of the motor, but vibration also relates to the various parts of the motor. Even the same type of motor, the vibration is also very different, which also increases the vibration of complexity.

Measurement and analysis of noise and vibration of the motor, the basis of its design is the collection and processing of motor noise and vibration signals, and therefore need to develop an efficient motor noise and vibration testing, treatment and diagnostic systems commonly used in the motor noise measurement tool is sound level meter, measuring the motor noise level and simple spectral analysis; motor vibration test commonly used pick-up count, or by a recorder records the acceleration sensor signal, the signal processing methods are usually based on spectral analysis of the complex Fourier transform processing is usually need to buy expensive equipment, such as the Danish company B\&K PULSE analysis system, Germany Muener BBM company BBMRAK system. domestic popularity of Beijing technology Co., Ltd. Vs302USB dual-channel acoustic analyzer, vibration and noise Oriental Institute of Technology INv3033063018 intelligent data acquisition and The signal processing analysis system

\section{Noise and Vibration Characteristics of the Motor}

Any mechanical vibration system is acoustic source. Motor noise is generated due to the vibration motor, so the motor noise analysis can be attributed to the vibration of the motor the noise of the motor is substantially involved in the overall effect of the motor, but also relates to vibration various components motor. The following were elaborated noise vibration motor vibration motor generally includes a stator, a rotor, base, and bearing vibration, special motors have different parts, so there are large differences in vibration motor can be summarized as figure 1 . 


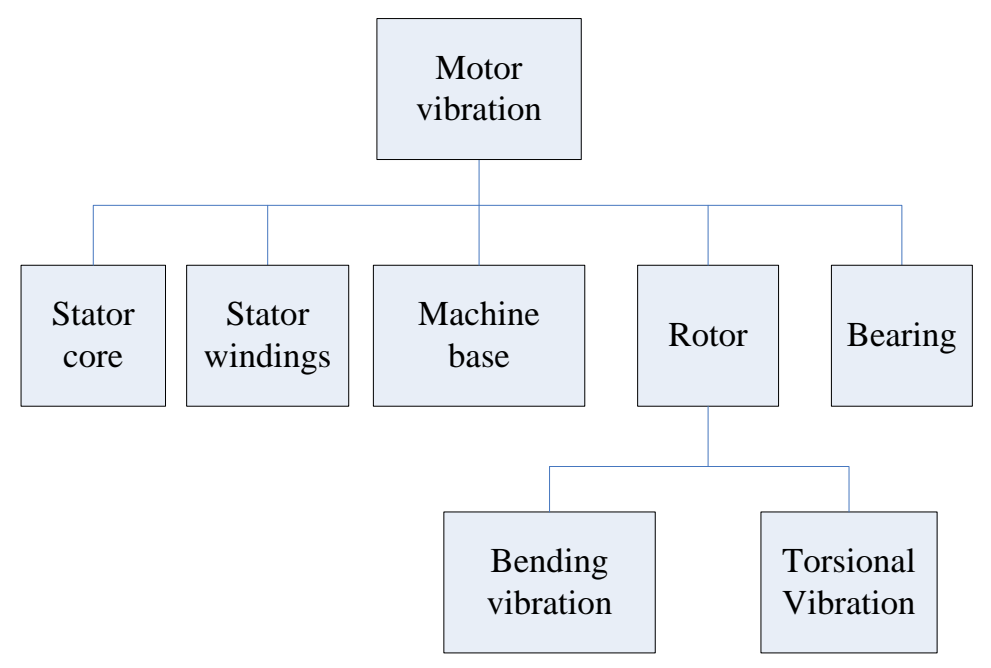

Fig.1 Motor vibration form

\section{Hardware of the System}

The hardware can be divided into sensors, signal conditioning circuits, and data acquisition card in three parts; software includes signal acquisition and storage, signal processing and fault diagnosis in part by vibration and sound sensors, signal conditioning circuits combined to give the motor data acquisition card. It has vibration and sound signals. Software with graphical programming software, Labview has prepared to give a good man-machine interface, easy to operate virtual instrument.

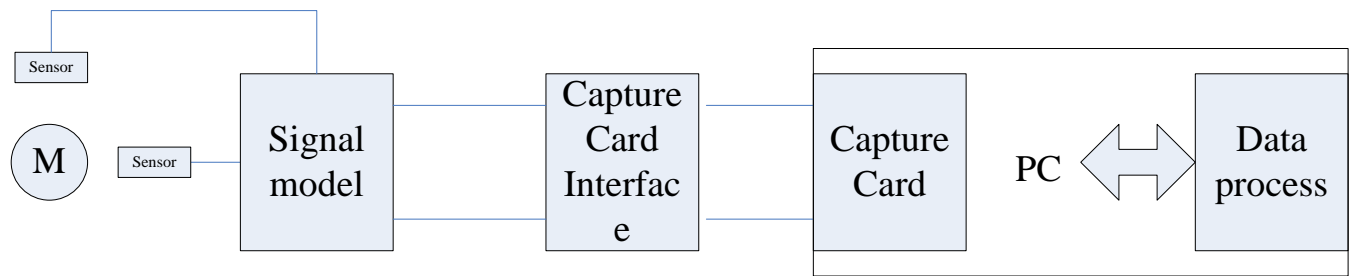

Fig. 2 Block diagram of the overall system

\section{Software of the System}

The software part of the system with the United States National Instruments Labview virtual instrument software with MATLAB call to complete the preparation, mainly divided into functional signal acquisition and preservation, signal analysis, troubleshooting, and print reports, etc. Since Labview own characteristics, making it easy modular program design, content can be individually designed for each debugging, greatly speeding up the design process of this software is mainly used in electrical fault diagnosis by collecting and saving signal from the system hardware to give the motor state data; then, the structure of the frame to get the motor state by a signal processing feature vector, for manual or intelligent diagnostic software diagnostic fault shown in Figure 3.

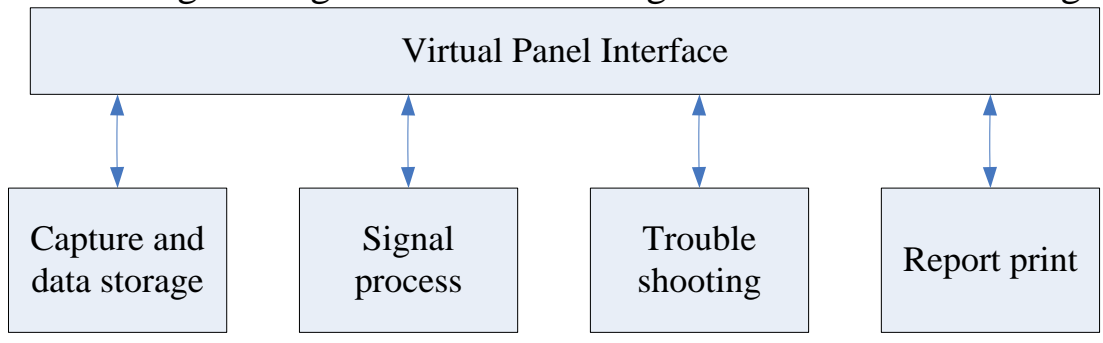

Fig.3 Software framework

Traditional instruments all software and measurement circuitry packaged together using the instrument front panel provides users with a limited set of functions. The system provides a virtual instrument is required to complete the measurement or control task of all software and hardware equipment, function entirely by user-defined, you can use the efficient and powerful software to 
customize the collection, analysis, storage, sharing, and display functions can be applied to achieve a different device or many applications, a device.

Compared with traditional instruments, virtual instrument hardware performance is better. Important concept of virtual instrument is driven by actual virtual instrumentation software and hardware device acceleration strategy. Virtual instrument system is software-based, so long as it is something that can be digitized, it can measure it.

Virtual instrument is fully compatible with traditional instruments, without exception, virtual instrumentation software typically provides libraries with common ordinary instrument buses (such as GPIB, serial and Ethernet) connected to the instrument drivers provide a high level and can be read function and instrument interface. All instrument drivers are designed for a particular model instrument designed to provide an interface to its unique properties.

Labview Development Platform. Labview program called Virtual Instrument (virtual instruments) program, referred to as a basic VL by the three parts: the front panel (panel), a block diagram of the program (Diagram Program) connection port and icon (Icon/Terminal).

Signal Analysis and Processing. Hardware system plus information obtained by data acquisition software are dynamic waveforms can represent various physical quantities, this system gathers the resulting signal is vibration acceleration and sound signals. They all have one and the same form of expression, that is, the physical quantity over time there are some changes the trajectory, with time as the abscissa, ordinate various physical quantities as to obtain a change in the graphics, is the waveform (signal) from these waveforms can get some physical information, such as amplitude, period, etc., but often very limited and insufficient because often composed of many dynamic waveform amplitude, frequency, phase relationship of the different waveform superposition mixed. To decompose various frequency components of the composite analog signals in the effective amount of the signal must be processed) signal processing. The most common approach is to decompose the dynamic waveform, and which consists of time as abscissa the time domain to the frequency converter on the horizontal axis of the frequency domain analysis, such as spectrum analysis, power spectral density function analysis. The signal processing system design is important module, the main application of the four methods, including fast Fourier analysis, power spectrum analysis function, further analysis of fractional fold spectrum analysis and wavelet analysis based on their results, and signal analysis Comparison of the calculated spectrum, to verify the correctness.

Fault Diagnosis. Fault diagnosis module with support vector machine implementation, in particular by Labview application MATLAB Script call MATLAB program LS a SV-Matlab, toolkit implementation. Support vector machine has been applied to many real-world problems, including text classification, image recognition biological sequence analysis and biological data mining, handwritten character recognition, etc. Although SVM achieved very good results in the classification of support, but is still relatively little application in motor failure diagnosis. It will support vector machine theory is introduced into the fault diagnosis in induction motors. Like SVM classification in other applications, SVM classifier as fault diagnosis, its design includes core selection and parameter optimization in two ways. in general, the choice of the actual nuclear on selecting a family having a core of several ultra-parameters, such as a Gaussian kernel parameters determined. after selecting the appropriate kernel, you need to determine the classification is to use the largest gap is the choice between a soft interval.

Integration of System Software. Software design using UI event loop programming mode user interface event loop mode is an effective way the user interacts with the Labview program processing, it can be used to detect the user to change the value of a button, move or click the mouse, press a keyboard, etc. Because of this model can accurately respond when an event occurs, and the rest in the middle of two events, changes in the value of the button without loop detection, you can save the system money.

Signal acquisition and signal processing with a vibration sensor in the following example, the description of the program flow. 
1) Connect the three-phase transformer and three-phase asynchronous motors, three-phase and three-phase power transformer is connected;

2) Closing the three-phase power switch, and gradually adjust the transformer to the rated voltage $380 \mathrm{~V}$; open vibration sensor ICP power switch, the establishment of an external hardware;

3) Run the application, enter the data acquisition interface; physical channel pre-set collection of A10, set the minimum and maximum values, sampling rate and number of samples;

4) Incoming signal analysis page, there are four displays waveform, are the fast Fourier transform, power spectrum analysis function, fractional times of spectral analysis and wavelet analysis on the left can be selected online analysis and offline analysis, online analysis represents analysis of the direct signal source is waveform acquisition, offline analysis indicates that the data source for the analysis of the saved data file to $380 \mathrm{~V}, 50 \mathrm{~Hz}$ three-phase power supply, the motor is normal, off-line analysis, for example, click on the button signal analysis.

\section{Conclusion}

This paper provides an overview of the basic concepts of the software block diagram of the system, including signal acquisition and preservation of four parts, signal processing, fault diagnosis and printouts, etc., then studied the various parts of the implementation issues. elaborated signal acquisition principle not discuss the accuracy of the block diagram of the signal acquisition and implementation. realized the whole file storage and file storage block, respectively, for data analysis and troubleshooting. study of four signal processing methods, including Fourier analysis, power spectrum analysis function, fractional times spectrum analysis and wavelet analysis, to achieve a block diagram of the signal processing method studied SVM principles and least squares support vector machine algorithm, discussed motor fault diagnostic process uncertainty and describes the algorithm of fault diagnosis.

\section{References}

[1] Aifat Siddique, GS. Yadava, Bhim Singh. A Review of Stator Fault Monitoring Techniques of Induction Motors. IEEE Transaction on Energy Converse,2005, 20(1): 245-259.

[2] S. Nandi and H. Toliyat, Condition Monitoring and Fault Diagnosis of Electrical Machines A Review.In Proceedings IEEE-IEMD Conference, Seattle,WA,1999:352-358.

[3] F. Filippetti, G. Franeesehini, C. Tassoni, P.Vas. Al Techniques in Induction Machines Diagnosis Including the Speed Ripple Effect. IEEE-IAS Annual Meeting conference, SanDiego, 1996:655-662.

[4] RYchetsky, M; Ortmaxm,s; Glesner, M. Support Vector Approaches for Engine Knock Detcetion. International Joint Conference on Neural Networks,Jul1999,Vol 12:969-974. 